Version pré-print de l'article paru dans Langue Française n 200 (2018) pp. 19-33.

\title{
Laurent Gosselin
}

\section{Résumé :}

Le conditionnel temporel subjectif et la possibilité prospective

Cet article traite de l'emploi dit «temporel subjectif» du conditionnel (ex. : «Il savait que je viendrais »). Il expose d'abord les difficultés auxquelles se trouve confrontée l'analyse de cet emploi au moyen du concept de possibilité prospective (analyse proposée par Gosselin 1999, et dans le cadre de la sémantique du temps ramifié par Martin 1981, Vuillaume 2001 et Bres 2009). Il propose ensuite, dans ce cadre, une analyse nouvelle de la possibilité prospective, qui répond aux difficultés signalées et fournit de nouvelles prédictions sur la sémantique des énoncés au conditionnel subjectif.

Mots-clés : conditionnel, possibilité prospective, temps ramifié, factivité, point de vue subjectif

\begin{abstract}
:
The "subjective temporal" use of conditional tense and prospective possibility

This paper deals with the so-called "subjective temporal" use of the conditional tense (eg "Il savait que je viendrais", "He knew that I would come"). First we will expose the difficulties to which is confronted the analysis of this use by means of the concept of prospective possibility (see Gosselin 1999, and in the framework of branching time semantics, Martin 1981, Vuillaume 2001 and Bres 2009). We then propose, in this framework, a new analysis of prospective possibility, which responds to the indicated difficulties and provides new predictions on the semantics of statements using "subjective temporal" conditional tense.
\end{abstract}

Keywords: conditional tense, prospective possibility, branching time, factivity, subjective point of view

\section{Le conditionnel temporel subjectif et la possibilité prospective}

\section{Introduction}

On distingue classiquement les emplois dits «temporels» des emplois dits «modaux» du conditionnel. Il s'agit là, au mieux, d'une commodité, car tous les emplois contiennent des composantes temporelle, aspectuelle et modale. Ainsi l'emploi «modal » de la forme simple $\mathrm{du}$ conditionnel («je serais riche, j'achèterais une voiture ») exclut-il que les procès soient localisés dans un passé révolu (contrainte temporelle). À l'inverse, les emplois «temporels » se répartissent en emplois «subjectif » et «objectif» (Nilsson-Ehle 1943, Bres 2012), ce qui constitue, à l'évidence, une distinction de nature modale. L'emploi dit «temporel subjectif » (Korzen et Nølke 2001: 129, Dodig 2018: 245-248), correspond à l'expression d'une 
ultériorité du moment de référence qui affecte le procès par rapport à un autre moment, situé dans le passé, et correspondant à l'expression d'un point de vue subjectif. Ce point de vue est exprimé de façon explicite dans la phrase elle-même par un verbe d'attitude propositionnelle ou par un verbe de communication, qui régit la subordonnée au conditionnel (ex. 1). Mais il peut aussi être récupérable dans le contexte de la phrase au conditionnel, qui est alors au style indirect libre (SIL) (cf. Nilsson-Ehle 1943 : 55, Azzopardi et Bres 2011 : 60), comme dans l'exemple (2) :

(1) Ils ajoutèrent même que, très vraisemblablement, ce serait dans la baie de Saint-Andrews que le commodore Dupont viendrait mouiller [...]. (J. Verne, Nord contre Sud)

(2) Tous se trouvaient maintenant réunis. Ces mauvais jours, on les passerait ensemble, avec plus de sécurité et surtout avec de moindres angoisses. (J. Verne, Nord contre Sud)

Dans le but de définir une valeur unique en langue du conditionnel, il a été proposé (Gosselin 1999, 2001) d'articuler la structure aspectuo-temporelle du conditionnel (comme ultérieur du passé), à la modalité du possible prospectif (Von Wright 1984'). Dans l'exemple (1), le point de référence correspondant au verbe de la principale ${ }^{2}$ opèrerait la coupure modale entre l'irrévocable (antérieur) et le possible (ultérieur). Dans le cadre de la logique du temps ramifié (Martin1981, Vuillaume 2001, Bres 2009), cela revient à dire que ce moment de référence constitue un point de ramification du temps, et que le procès au conditionnel se situe sur au moins une des branches, correspondant aux suites possibles de ce point de ramification. Cette hypothèse, qui permet de rendre compte, au moyen d'une valeur unique, à la fois des emplois «temporels » et des emplois «modaux»- et tout particulièrement de l'emploi hypothétique ${ }^{3}$ - se heurte cependant à un ensemble de difficultés.

S'il est vrai que dans

(3) Luc croyait / disait / pensait que Marie reviendrait

la proposition exprimée par la complétive paraît effectivement conçue comme simplement possible (au sens ou sa valeur de vérité reste indéterminée, cf. Korzen et Nølke1990 : 274), cela semble dû bien davantage au verbe de la principale, qui est non factif, qu'au conditionnel, car on observe la même indétermination avec les autres temps verbaux de l'indicatif :

(4a) Luc croyait / disait / pensait que Marie l'aimait.

(4b) À vous voir marcher comme ça, j'ai cru que vous étiez en train d'accoucher et que vous perdriez les eaux sur ma banquette arrière. (G. Leroy, Alabama song).

De plus, avec un verbe factif (savoir, ignorer) ou contrefactif (s'imaginer, se figurer) la valeur de la proposition n'est plus indéterminée, mais présupposée vraie ou fausse, au conditionnel comme aux autres temps verbaux :

(5a) Luc savait / ignorait / se doutait que Marie reviendrait.

(5b) Luc s'imaginait / se figurait que Marie reviendrait.

Enfin, si la proposition au conditionnel était, dans ces tours, simplement possible, on serait en droit d'attendre que le verbe de la principale porte sur cette modalité du possible, comme dans l'exemple suivant où la possibilité est exprimée par pouvoir :

(6) Luc croyait qu'il pouvait traverser à pied sec ( $~ q u ' i l$ était possible qu'il traverse à pied sec).

\footnotetext{
${ }^{1}$ La possibilité prospective appartient aux modalités diachroniques de Von Wright (1984), des modalités affectées d'un indice temporel différent de celui qui est attribué au contenu propositionnel sur lequel elles portent.

${ }^{2}$ Dans la perspective dialogique de Bres (2009), il s'agit d'un moment d'énonciation secondaire.

${ }^{3}$ Gosselin (2001) montre qu'elle permet aussi d'expliquer certaines analogies de fonctionnement entre le conditionnel et le subjonctif.
} 
Or il n'en est rien. (7b) ne constitue pas une glose acceptable de (7a) (ce n'en est qu'une implication) :

(7a) Luc croyait que Marie traverserait à pied sec.

(7b) Luc croyait qu'il était possible que Marie traverse à pied sec.

Pour résoudre ces difficultés, nous nous proposons d'analyser plus précisément la notion de possibilité prospective et de l'articuler à la fois à la structure aspectuo-temporelle du conditionnel et à la structure modale de ces tours. Mais il convient préalablement de mieux définir l'emploi « temporel subjectif » que nous étudions.

\section{Définition de l'emploi «temporel subjectif »}

Dans son emploi «temporel », le conditionnel marque l'ultériorité dans le passé. Cette valeur sémantique se laisse directement articuler à la morphologie (Wilmet 2001, Gosselin 2001, 2017). On admet, en effet, que ce temps verbal comporte, en synchronie, une flexion double, composée d'un élément [r] indiquant l'ultériorité (cf. Imbs 1960: 61, Azzopardi et Bres 2011), que l'on retrouve également dans la formation du futur, et d'un élément correspondant aux flexions de l'imparfait. Par exemple, dans «Je mangerais", on reconnaît le [r] d'ultériorité associé au [ع] de l'imparfait (Touratier $1996: 38$ ).

Dans le modèle SdT (Gosselin 1996, 20174), deux intervalles de référence sont associés au conditionnel : respectivement $\mathrm{R}_{2}$ marqué par [r], et $\mathrm{R}_{1}$ marqué par la flexion de l'imparfait. La valeur d'ultériorité de [r] localise $R_{2}$ comme postérieur à $R_{1}$, et indique que $R_{2}$ porte sur $E$ selon une visée aspectuelle sous-déterminée. D'où la formule : $\mathrm{R}_{1}<\mathrm{R}_{2} ; \mathrm{R}_{2} \subseteq \mathrm{E}$. La valeur de IMP indique que $R_{1}$ est situé dans le passé $: R_{1}<S$. Dans les complétives, $R_{1}$ coïncide avec le $\mathrm{R}$ de la principale :

(8) Il disait qu'il viendrait.

$$
\begin{aligned}
& \mathrm{R}_{1}<\mathrm{R}_{2} ; \mathrm{R}_{2} \subseteq \mathrm{E} \\
& \mathrm{R}_{1}<\mathrm{S} \\
& \mathrm{R} 1=\mathrm{R}_{\text {princ }}
\end{aligned}
$$

la position de $\mathrm{R}_{2}$ et $\mathrm{E}$ relativement à $\mathrm{S}$ n'est pas contrainte (le procès «il-venir » peut aussi bien être envisagé dans le passé, le présent ou l'avenir ; Gosselin 2001 : 59).

L'emploi temporel «subjectif » se distingue de l'emploi «objectif » (Bres 2012, Dodig 2018 : 245-254). Si l'emploi « objectif » implique systématiquement la réalisation du procès, il n'en va pas de même pour l'emploi «subjectif». Toutefois, ce critère est fragile, car lorsque le verbe recteur est factif, comme en (5), la vérité de la proposition au conditionnel «subjectif » est présupposée. Tout en tenant compte des observations de Bres, Diwersy et Luxardo (ce volume), nous maintenons qu'il s'agit, dans ce type d'exemple, de conditionnel « subjectif » dans la mesure où les verbes en question, de même que les subjectifs non-factifs, construisent un contexte opaque, i.e. un contexte dans lequel les expressions référentiellement équivalentes ne sont pas substituables salva veritate:

(9a) Edipe croyait / savait / était conscient / avait décidé / dit qu'il épouserait Jocaste / ? sa mère.

(9b) Edipe ignorait / ne savait pas / ne pouvait imaginer qu'il épouserait sa mère / ? Jocaste.

Certes un verbe comme ignorer n'exprime pas une prise de position d'un énonciateur différent du locuteur, mais envisage tout de même le contenu de la complétive à partir du point de vue de cet énonciateur (d'où l'opacité).

Au plan syntaxique, le conditionnel temporel subjectif peut se manifester dans une subordonnée : a) complétive objet d'un verbe d'opinion ou de communication (marquant la

\footnotetext{
${ }^{4}$ Le modèle SdT (Sémantique de la Temporalité) est de type néo-reichenbachien. S, R et $\mathrm{E}$ désignent respectivement le moment d'énonciation, le moment de référence, et celui du procès. Ces moments sont systématiquement conçus comme des intervalles.
} 
production ou la réception), ou encore d'une construction impersonnelle (il était certain / probable que), b) interrogative indirecte, c) relative, d) circonstancielle.

Il peut aussi apparaître dans une indépendante ou une principale, avec effet de discours rapporté au SIL. Le point de vue subjectif est alors construit par l'interprétation du contexte et peut être glosé au moyen d'un verbe recteur qui le restitue de façon explicite :

(10) J'avais encore un espoir, c'est qu'une fois arrivés au fond du cratère, il serait impossible, faute de galerie, de descendre plus profondément[...]. [ $\approx$ j'espérais qu'il serait impossible de ...] (J. Verne, Voyage au centre de la terre)

\section{La structure modale de ces constructions}

Nous reprenons, dans ses grandes lignes, l'analyse modale de ces constructions présentée dans Gosselin (2014). Le point de vue subjectif correspond à une modalité extrinsèque (notée $\operatorname{Mod}_{\mathrm{i}}$ ) qui porte sur la modalité intrinsèque au prédicat (notée $\operatorname{Mod}_{\mathrm{j}}$ ), dans une structure modale générale :

(11) $\operatorname{Mod}_{\mathrm{i}}\left(\operatorname{Mod}_{\mathrm{j}}(\operatorname{Pred}(\arg )).\right)$

Par exemple, dans la phrase

(12) Luc savait que Marie reviendrait

le point de vue subjectif $\left(\operatorname{Mod}_{\mathrm{i}}\right)$ est explicitement marqué par le verbe épistémique savoir, tandis que le prédicat (revenir) est intrinsèquement porteur d'une modalité aléthique $\left(\mathrm{Mod}_{\mathrm{j}}\right)$. Cette modalité intrinsèque serait axiologique en (13) :

(13) Luc savait que Marie serait courageuse.

La validation de la modalité intrinsèque correspond à la réalisation du procès (revenir, être courageux).

Le verbe recteur peut être purement modal (ex. savoir, croire, penser...) ou partiellement modal, en particulier dans le cas des verbes de communication, qui marquent l'expression d'une pensée (dire, écrire, annoncer...) ou sa réception (lire, comprendre, apprendre...).

La modalité extrinsèque exprimée par ce verbe recteur ou reconstituée à partir du contexte dans le cas du discours rapporté au SIL, qui correspond au point de vue subjectif, peut être épistémique (croire, savoir) ou mixte (espérer: épistémique et boulique, cf. Gosselin 2010). Il est à noter que l'emploi du SIL permet de faire dépendre les procès au conditionnel de modalités extrinsèques déontiques ou appréciatives, qui, si elles étaient exprimées par un verbe recteur, exigeraient le subjonctif ou l'infinitif :

(14) McKenna énuméra alors les mesures de sécurité à prendre. Tous les accès de la ville seraient contrôlés. [ $\approx$ il dit de contrôler tous les accès / que tous les accès soient contrôlés] (J. Dicker, $L a$ disparition de Stéphanie Mailer)

Il est possible d'insérer une autre modalité extrinsèque entre $\operatorname{Mod}_{\mathrm{i}}$ et $\operatorname{Mod}_{\mathrm{j}}$, selon la structure :

(15a) $\operatorname{Mod}_{\mathrm{i} 1}\left(\operatorname{Mod}_{\mathrm{i} 2}\left(\operatorname{Mod}_{\mathrm{j}}(\operatorname{Pred}(\arg )).\right)\right)$

(15b) Luc pensait ( $\operatorname{Mod}_{\mathrm{i} 1}$ : épistémique, probable) qu'il lui serait possible ( $\operatorname{Mod}_{\mathrm{i} 2}$ aléthique, possible) de franchir le fossé (Mod $\mathrm{j}$ : aléthique).

En revanche, la présence d'un adverbe épistémique, fréquente dans ces tours, ne déclenche pas systématiquement la construction d'une seconde modalité extrinsèque. Lorsqu'un verbe recteur épistémique (croire, savoir, penser...) se combine avec un adverbe épistémique (peutêtre, certainement, sans doute...) dans la complétive, ce dernier n'ajoute pas une seconde modalité épistémique, mais sert à préciser le degré de croyance exprimé :

(16) [...] l'ingénieur pensait qu'il serait peut-être possible d'utiliser cette chute et de lui emprunter sa force [...]. (J. Verne, L'Île mystérieuse). 
Il en va de même lorsque cette modalité épistémique est exprimée par un verbe de communication (ex. 1 supra), ou construite par l'interprétation du SIL (17) :

(17) Le moment arrivé, il dit adieu à sa femme, à sa fille, à Miss Alice [...]. Très certainement, il serait de retour le jour même. (J. Verne, Nord contre Sud)

Dans la structure générale (11), telle qu'elle est analysée dans Gosselin (2014) et illustrée par l'exemple (12), le verbe recteur sert à indiquer la catégorie et la valeur modale (ex. épistémique certain), de $\mathrm{Mod}_{\mathrm{i}}$, mais aussi le degré de prise en charge de $\mathrm{Mod}_{\mathrm{j}}$ par le locuteur. S'il implique la prise en charge (savoir, ignorer, se douter, révéler), l'interprétation sera factive (cf. supra, ex. 5a); s'il marque le refus de prise en charge (s'imaginer, se figurer), elle sera contrefactive (ex. 5b); s'il n'exprime ni l'un ni l'autre (croire, penser, dire), elle sera simplement non factive (ex. 4a, b).

La question se pose alors, plus précisément, de savoir quelles sont les contraintes exercées par le conditionnel dans cette structure modale. Il affecte manifestement deux paramètres de $\operatorname{Mod}_{\mathrm{j}}$ (ou de $\operatorname{Mod}_{\mathrm{i} 2}$, le cas échéant) : les indices temporels (puisqu'il marque l'ultériorité du procès par rapport au point de vue subjectif) et la force de validation, dont les auteurs cités supra font l'hypothèse qu'elle serait simplement possible. C'est la conjonction de ces deux instructions (ultériorité et possibilité) qui fait du conditionnel dans ces tours un marqueur de possibilité prospective, un marqueur de ramification temporelle dans le cadre d'une sémantique du temps ramifié.

Et l'on retrouve alors les difficultés signalées dans l'introduction : dans l'exemple (12), le verbe savoir exprime la certitude et la factivité, mais cette certitude et cette factivité ne portent pas sur la simple possibilité du procès, mais bien sur sa réalisation effective (sur la validation de la prédication). Comment rendre compte de ce fonctionnement dans le cadre proposé ? Deux solutions paraissent envisageables :

a) abandonner l'idée selon laquelle le conditionnel marquerait la possibilité prospective et renoncer à identifier une valeur unique en langue pour ce temps verbal ;

b) proposer une analyse nouvelle de la possibilité prospective qui permette de concilier des exigences apparemment contradictoires.

C'est cette deuxième solution qui va être adoptée et développée ici.

\section{La possibilité prospective}

La réflexion sur la possibilité prospective remonte à Aristote, qui l'envisage selon trois points de vues distincts, mais interconnectés : logique, pratique et ontologique. Dans une perspective logique et énonciative, l'analyse fameuse des propositions portant sur l'avenir (ex. « il y aura une bataille navale demain »), développée au chapitre 9 du traité De l'interprétation, établit que ces propositions font exception au principe de bivalence (Vuillemin 1984 : 161 sq.), dans la mesure où elles ne sont, au moment d'énonciation, ni vraies ni fausses. En revanche, au moment futur évoqué, elles seront nécessairement soit vraies, soit fausses. De là une structure modale du temps qui est asymétrique : le présent et le passé sont irrévocables (les valeurs de vérité des propositions au présent et au passé sont déterminées), tandis que l'avenir est le domaine des possibles (la valeur de vérité des propositions qui le concernent reste provisoirement indéterminée).

Il est montré dans l'Ethique à Nicomaque (VI, 2, 1139b 6-11) que cette asymétrie modale concerne fondamentalement le temps pratique, de la délibération, de la décision et de l'action. C'est parce qu'on ne peut délibérer, décider et agir que sur les événements futurs, que l'on considère le passé comme irrévocable et l'avenir comme ouvert, simplement possible.

Enfin, dans une perspective ontologique (Métaphysique IX), Aristote envisage le mode d'être de ces événements futurs, et met en place le couple de notions indissociables que sont la puissance et l'acte. Avant d'avoir lieu, un procès (état ou événement) n'existe qu'en 
puissance, il n'est en acte qu'à partir du moment où il se réalise (il s'actualise). De sorte qu'au moment d'énonciation, les procès futurs ne sont, actuellement, qu'en puissance, tandis que ce qui est envisagé, comme pure virtualité, dans l'avenir, c'est l'actualisation de cette puissance (la réalisation des procès).

Transposée au plan des modalités linguistiques, cette conception aristotélicienne de la possibilité prospective, nous a conduit (cf. Gosselin $2010: 223-225)$ à poser que :

a) la possibilité prospective est une valeur transmodale, au sens où elle concerne toutes les catégories modales (aléthique, épistémique, appréciative, etc.) qui réside dans l'indétermination provisoire de la force de validation ;

b) elle est composée de deux valeurs distinctes, mais strictement articulées : 1) une valeur actuelle (i.e. qui vaut au moment pris pour repère d'actualité) qui est indéterminée (la validation de la prédication n'est ni positive ni négative) et 2) une valeur virtuelle (qui vaut au moment où le procès est censé se produire), qui est déterminée (positivement ou négativement, selon la valeur du prédicat et des éventuels opérateurs de négation qui l'affectent).

Prenons un exemple au futur simple :

(18) Luc reviendra la semaine prochaine.

Cette analyse implique qu'au moment de l'énonciation, la validation de la prédication ( $L u c$ revenir la semaine prochaine) est actuellement indéterminée - le procès n'est alors qu'en puissance - tandis qu'elle est envisagée comme virtuellement déterminée positivement - le procès est envisagé dans son actualisation - pour la période correspondant à la semaine prochaine. Or si on inscrit cette proposition dans une complétive régie par le verbe savoir, par exemple :

(19) Marie sait que Luc reviendra la semaine prochaine

on s'aperçoit que :

1) la modalité épistémique de croyance positive marquée par savoir $\left(\operatorname{Mod}_{\mathrm{i}}\right)$ porte exclusivement sur la valeur virtuelle déterminée de $\operatorname{Mod}_{\mathrm{j}}\left(\right.$ notée $\left.\operatorname{Mod}_{\mathrm{j} 2}\right)$ : Marie croit à la réalisation future du procès, et pas seulement à sa possibilité ;

2) la factivité - i.e. la prise en charge de $\operatorname{Mod}_{\mathrm{j}}$ par le locuteur - ne porte, elle aussi, que sur la valeur virtuelle, déterminée, de $\operatorname{Mod}_{\mathrm{j}}: \operatorname{Mod}_{\mathrm{j} 2}$.

Qu'est-ce qui atteste dès lors de la pertinence d'attribuer une valeur actuelle indéterminée à $\operatorname{Mod}_{\mathrm{j}}\left(\right.$ notée $\left.\operatorname{Mod}_{\mathrm{j} 1}\right)$ ? C'est la possibilité de substituer au verbe savoir un verbe exprimant une délibération, une décision, qui indique explicitement le choix d'une possibilité parmi d'autres, comme dans (20a), que l'on opposera à (20b) :

(20a) Il est décidé / convenu/ Marie décide que Luc reviendra la semaine prochaine.

(20b) ?? Il est décidé / convenu/ Marie décide que Luc est revenu la semaine dernière.

En (20a), la décision porte sur la valeur virtuelle, déterminée $\left(\operatorname{Mod}_{\mathrm{j} 2}\right)$, mais présuppose sa valeur actuelle indéterminée $\left(\operatorname{Mod}_{j 1}\right)$, selon une structure du type :

(21) Modi $\left(\operatorname{Mod}_{\mathrm{j} 2}\right.$ (Pred. (arg.))) \& $\operatorname{Mod}_{\mathrm{j} 1}$ (Pred. (arg.)).

Dans le cadre de la sémantique du temps ramifié, cette double valeur de la possibilité prospective correspond simplement au fait que le procès est envisagé comme réalisé (valeur virtuelle déterminée) sur une (au moins) des suites possibles du temps, considérée au moment actuel. Le point de vue subjectif, comme la factivité, portent sur cette réalisation du procès. Soit sous forme schématique, la structure associée à (19) : 


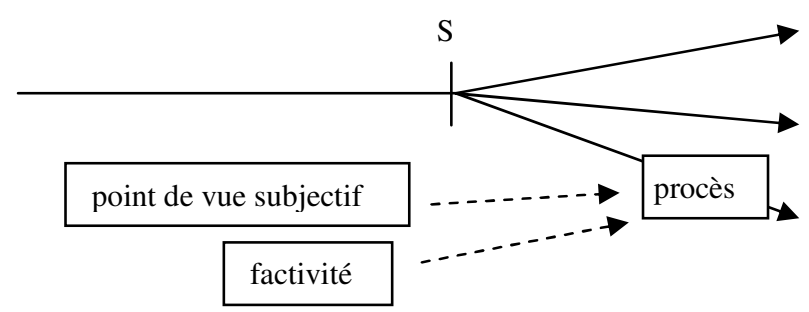

Figure 1 : structure de l'ex. (19)

Reste qu'au conditionnel temporel subjectif, cette possibilité prospective est envisagée à partir d'un point de vue subjectif $\left(\mathrm{Mod}_{\mathrm{i}}\right)$ lui-même situé dans le passé, et que les procès qu'elle concerne peuvent eux-mêmes être localisés dans le passé, le présent ou l'avenir. Dès lors, la coupure modale ne va plus - ou plus seulement - passer par le moment d'énonciation.

\section{Analyses modale et aspectuo-temporelle du conditionnel 5.1 La coupure modale}

Pour Aristote, c'est le moment présent qui opère la coupure modale, entre l'irrévocable (passé) et le possible (futur). Selon la théorie du temps ramifié, tout moment du temps constitue un point de ramification, entre ce qui est antérieur (linéaire, irrévocable) et ce qui est ultérieur (ramifié, possible) (Thomason 1970). Mais le moment présent jouit tout de même d'un statut particulier, car parmi les suites possibles issues de ramifications situées dans le passé, une seule s'est réalisée, alors que les autres ne sont plus possibles. À l'inverse, celles qui sont issues de ramifications situées dans le présent ou l'avenir sont toutes encore possibles (Martinez 2011 : 272-273). Graphiquement, les suites qui ne sont plus possibles sont notées en pointillés, les autres par des flèches pleines :

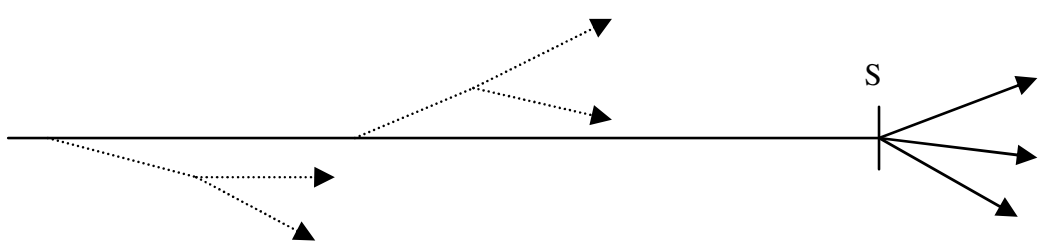

Figure 2 : structure du temps ramifié

Qu'en est-il dans le champ de la sémantique linguistique ? Dowty (1979) a montré qu'il pouvait être utile de considérer un point de ramification différent du moment présent, pour rendre compte du «paradoxe imperfectif», qui résulte de l'interaction de l'aspect et de la modalité. Ainsi, de l'énoncé

(22) Il traversait le carrefour

on ne peut conclure que le procès a été conduit jusqu'à son terme (un empêchement a pu survenir). La visée aspectuelle imperfective introduit un point de ramification au milieu de l'intervalle du procès, qui situe le début du procès dans l'irrévocable et sa fin dans le possible. Cette analyse a été généralisée par Vet (1981) qui considère que dans le système de Reichenbach, tout point $\mathrm{R}$ constitue un point de ramification, et par Gosselin (2005), qui pose l'existence de deux types de modalités en relation avec le temps et l'aspect: les modalités temporelles qui se construisent relativement à $\mathrm{S}$, et les modalités aspectuelles qui se définissent par rapport à $\mathrm{R}$ (ou, plus exactement, par rapport aux bornes finales de $\mathrm{S}$ et $\mathrm{R}$ qui sont des intervalles). Si bien que, dans un même énoncé, un procès pourra être présenté à la fois comme temporellement irrévocable et comme aspectuellement possible (partiellement ou totalement), ou - inversement - comme temporellement possible et aspectuellement 
irrévocable. Le contraste entre ces différentes valeurs modales donne lieu à des effets de sens particuliers. Ainsi, à la lecture de l'énoncé :

(23) Tard dans la nuit, à une date lointaine [...], je traversais la place des Pyramides vers la Concorde quand une voiture a surgi de l'ombre. (Modiano, Accident nocturne, phrase incipit).

on comprend que le procès traverser la place, étant passé (à une date lointaine), est temporellement dans l'irrévocable. Toutefois, comme il est présenté sous un aspect inaccompli, sa fin reste dans le domaine du possible. L'effet produit est celui d'un dévoilement progressif du cours des événements. Seule la lecture de la suite du texte permet de considérer l'achèvement du procès traverser la place comme une possibilité qui ne s'est pas réalisée (et qui ne peut plus l'être), selon une structure du type :

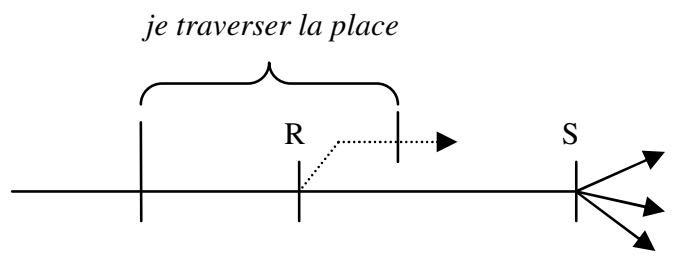

Figure 3 : structure de l'ex. (23)

Cette analyse a été étendue aux constructions périphrastiques complexes (Gosselin 2013), dans lesquelles c'est le moment de référence correspondant à l'élément verbal porteur d'une flexion personnelle qui opère la coupure modale aspectuelle. Par exemple, dans :

(24) On se disposa à achever de frapper un grand coup afin de couper le mal dans sa racine. (E.-L. Lamothe-Langon, Mémoires de Louis XVIII, 1832)

la coupure modale aspectuelle passe par la phase préparatoire (se disposa) à la phase finale (achever) du procès frapper un grand coup; de sorte que l'on ignore, provisoirement, si le procès lui-même a été conduit jusqu'à son terme.

La coupure modale temporelle coïncide avec $\mathrm{S}$; la coupure modale aspectuelle avec $\mathrm{R}$. Mais que se passe-t-il dans le cas du conditionnel, qui met en œuvre deux moments de référence, $\mathrm{R}_{1}$ et $\mathrm{R}_{2}$ ?

\subsection{Le conditionnel}

Nous avons avancé, dans Gosselin (2001), que la coupure modale aspectuelle était opérée par $\mathrm{R}_{1}$, le moment de référence antérieur, situé dans le passé et qui, dans l'emploi étudié ici coïncide avec celui du point de vue subjectif exprimé par le verbe de la principale ou récupéré dans le contexte (voir la structure de l'ex. 8 supra). C'est pourquoi le procès au conditionnel est simplement possible. En faveur de cette analyse, on peut avancer, comme pour le futur simple (ex. 20a), la possibilité d'introduire un procès au conditionnel dans une complétive régie par les verbes décider ou convenir, qui présupposent la possibilité prospective du procès :

(25) Il fut décidé entre les trois voyageurs qu'ils accosteraient la terre au premier emplacement favorable. (J. Verne, Cinq semaines en ballon)

Or nous avons proposé à la section précédente (ex. 21) de décomposer cette possibilité prospective en deux valeurs modales : une valeur actuelle indéterminée (notée $\operatorname{Mod}_{j 1}$ ) et une valeur virtuelle déterminée $\left(\operatorname{Mod}_{\mathrm{j} 2}\right)$ sur laquelle portent la modalité extrinsèque $\left(\operatorname{Mod}_{\mathrm{i}}\right)$ et la factivité, le cas échéant. Nous pouvons désormais affiner notre analyse modale du conditionnel, en avançant de nouvelles hypothèses : 
a) $R_{1}$ et $R_{2}$ opèrent deux coupures modales (ils constituent deux points de ramification), telles que la coupure modale marquée par $\mathrm{R}_{2}$ s'inscrit sur une des branches résultant de la ramification opérée par $\mathrm{R}_{1}$ selon un schéma du type :

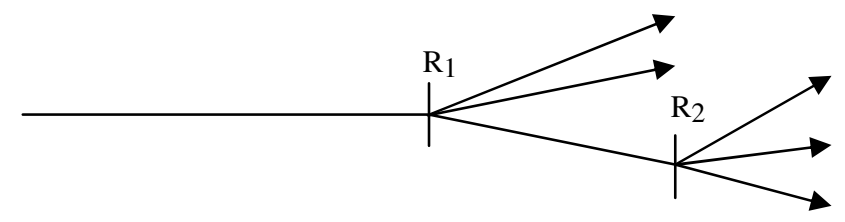

Figure 4 : structure du temps ramifié au conditionnel temporel subjectif

b) La valeur actuelle, indéterminée, de la modalité intrinsèque $\left(\operatorname{Mod}_{j 1}\right)$ se définit par rapport à $\mathrm{R}_{1}$ : ce qui précède est irrévocable, ce qui suit est simplement possible.

c) La valeur virtuelle, déterminée $\left(\operatorname{Mod}_{j 2}\right)$, se définit relativement à $R_{2}$ : seule la portion qui précède $\mathrm{R}_{2}$ est, virtuellement, déterminée ; ce qui suit reste indéterminé.

d) Comme la modalité extrinsèque ainsi que la factivité ne portent que sur la valeur déterminée de la modalité intrinsèque, elles n'affectent $\operatorname{Mod}_{\mathrm{j} 2}$ que jusqu'à $\mathrm{R}_{2}$; ce qui est audelà reste hors de portée de la modalité intrinsèque et de la factivité.

Appliquons ce dispositif explicatif à quelques exemples construits (qui nous permettent de contrôler tous les paramètres) :

(26a) Vendredi dernier, Marie pensait qu'hier midi, Luc se préparerait à / disposerait à / serait sur le point de faire les courses.

(26b) Vendredi dernier, Marie pensait qu'hier midi, Luc serait en train de faire les courses.

(26c) Vendredi dernier, Marie pensait qu'hier midi, Luc aurait fait les courses / rentrerait de faire les courses.

(27a) Vendredi dernier, Marie savait qu'hier midi, Luc se préparerait à / disposerait à / serait sur le point de faire les courses.

(27b) Vendredi dernier, Marie savait qu'hier midi, Luc serait en train de faire les courses.

(27c) Vendredi dernier, Marie savait qu'hier midi, Luc aurait fait les courses / rentrerait de faire les courses.

(28a) Vendredi dernier, Marie ignorait qu'hier midi, Luc se préparerait à / disposerai à / serait sur le point de faire les courses.

(28b) Vendredi dernier, Marie ignorait qu'hier midi, Luc serait en train de faire les courses.

(28c) Vendredi dernier, Marie ignorait qu'hier midi, Luc aurait fait les courses / rentrerait de faire les courses.

Au plan des modalités temporelles, tous les procès sont localisés dans le passé (hier midi) et donc dans l'irrévocable (dans ce qui a été actualisé ou non). Mais au plan des modalités aspectuelles, les situations diffèrent. Dans tous les cas, $\mathrm{R}_{1}$ coïncide avec le $\mathrm{R}$ de la modalité extrinsèque (le point de vue subjectif), de sorte que le procès (Luc faire des courses) est situé dans le domaine de la possibilité prospective. À ce moment-là (vendredi dernier), Luc pouvait encore décider ou non de faire les courses hier midi. Toutefois, comme la possibilité prospective se compose d'une valeur actuelle (indéterminée) et d'une valeur virtuelle (déterminée), et que la modalité extrinsèque ainsi que la factivité portent exclusivement sur cette valeur déterminée, on s'attend à ce que les verbes exprimant une croyance positive (penser, savoir) indiquent que Marie croyait à la réalisation du procès, et que les verbes factifs (savoir, ignorer) marquent la prise en charge par le locuteur de cette réalisation du procès. Mais ce serait sans compter avec la coupure modale secondaire, opérée par $\mathrm{R}_{2}$ : la valeur virtuelle de la possibilité prospective n'est déterminée que jusqu'à $\mathrm{R}_{2}$, qui est marquée par la flexion personnelle (se disposerait, serait en train, aurait...). De sorte que la croyance positive (penser, savoir) ne porte que sur la réalisation de la phase préparatoire du procès en (26a, $27 a)$, va jusqu'à sa phase médiane en (26b, 27b), et jusqu'à sa phase résultante en (26c, 27c). De même, la factivité (savoir, ignorer) implique respectivement la prise en charge par le 
locuteur de la validation des phases préparatoire (27a, 28a), médiane (27b, 28b), et résultante $(27 \mathrm{c}, 28 \mathrm{c})$ du procès. Si l'on souhaite extraire des informations sur la réalisation effective des procès, à partir de ces exemples, on déduira du dispositif mis en œuvre, que seuls $(27 \mathrm{c}, 28 \mathrm{c})$ impliquent que le procès est allé jusqu'à son terme, et que seuls $(27 b, c)$ et $(28 b, c)$ impliquent que le procès a eu au moins un début de réalisation.

$\mathrm{Si}$, de plus, on tient compte des modalités temporelles (définies relativement à $\mathrm{S}$ ), et si l'on sait, par ailleurs, que Luc a finalement renoncé à faire les courses, on illustrera (27a) par la figure 5 :

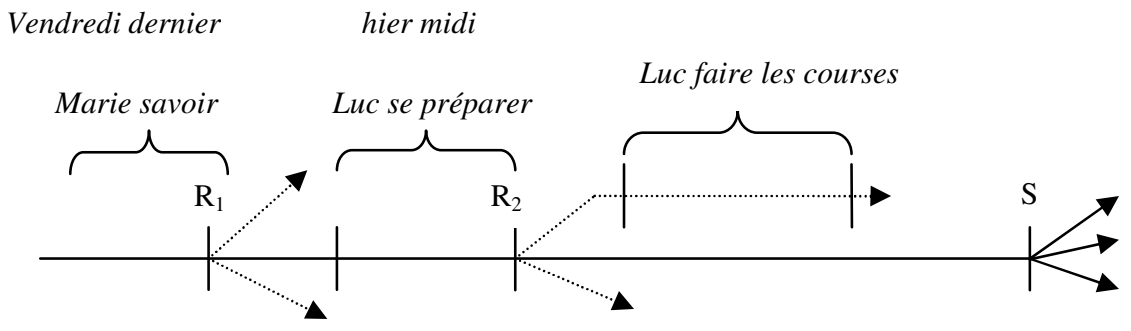

Figure 5 : structure de l'ex. (27a)

Ce dispositif offre l'avantage de maintenir une valeur en langue unique pour le conditionnel qui rend compte à la fois de l'emploi temporel subjectif et de l'emploi hypothétique, mais aussi, de ce fait, des emplois qui sont simultanément temporel et hypothétique :

(29) Il lui envoya plusieurs messagers pour le prier de venir le rejoindre sans retard à Castle-House. Là, on serait relativement en sûreté, et s'il fallait chercher une autre retraite, s'il fallait s'enfoncer dans l'intérieur du pays [...], il serait plus facile de le faire. (J. Verne, Nord contre Sud)

Les subordonnées hypothétiques expriment alors des conditions, des restrictions (au sens de Kratzer 1986), sur les suites possibles à prendre en compte: on ne retient que celles dans lesquelles les propositions exprimées par les subordonnées sont validées.

\section{Conclusion}

Nous avons proposé un dispositif explicatif, fondé sur la structure modale des énoncés au conditionnel temporel subjectif et sur une analyse nouvelle de la possibilité prospective articulée à la structure aspectuo-temporelle du conditionnel, qui permet de résoudre les difficultés signalées dans l'introduction, de maintenir une analyse monosémique en langue du conditionnel, et de faire de nouvelles prédictions sur l'interprétation des énoncés au conditionnel temporel subjectif.

Cette analyse proprement linguistique est utilisable pour l'étude des textes narratifs. Le conditionnel temporel subjectif permet de présenter des possibilités prospectives passées, au moment où elles n'étaient que de pures éventualités. L'un des intérêts de cet emploi pour le récit est d'exprimer des états et des processus mentaux des personnages, qui sont spécifiquement orientés vers des possibilités prospectives, comme l'attente, la peur, l'espoir, le désir, mais aussi les prédictions, les projets et les planifications. Le roman de J. Verne, Nord contre Sud, fait une utilisation particulièrement massive du conditionnel temporel subjectif, pour exprimer, et faire partager au lecteur le fait que les personnages éprouvent tour à tour, tout au long du roman, la crainte et l'espoir relativement à des événements ultérieurs, simplement possibles, et se livrent à des conjectures, des projets, que la suite du texte viendra confirmer ou infirmer.

\section{Références}

Aristote, éd. 1977, De l'interprétation, trad. J. Tricot, Paris, Vrin. 
Aristote, éd. 1981, La Métaphysique, trad. J. Tricot, Paris, Vrin.

Aristote, éd. 1983, Ethique à Nicomaque, trad. J. Tricot, Paris, Vrin.

Azzopardi, S. et Bres, J., 2011, Temps verbal et énonciation. Le conditionnel et le futur en français : l'un est dialogique, l'autre pas (souvent), Cahiers de praxématique, 56, 53-76.

Bres, J., 2009, Dialogisme et temps verbaux de l'indicatif, Langue française 163, 21-39.

Bres, J., 2012, Conditionnel et ultériorité dans le PASSÉ : de la subjectivité à l'objectivité, Actes du CMLF, $1719-1730$.

Dodig, M., 2018, Le conditionnel français et ses équivalents sémantiques en serbe. Etude comparative entre le conditionnel et le potentiel serbe, Thèse de l'Université Paul Valéry Montpellier 3.

Dowty, D., 1979, Word Meaning and Montague Grammar, Dordrecht, Reidel.

Gosselin, L., 1999, Les valeurs de l'imparfait et du conditionnel dans les systèmes hypothétiques, Cahiers Chronos 4 : 29-51.

Gosselin, L., 2001, Relations temporelles et modales dans le conditionnel journalistique, in Dendale P. et Tasmowski L. (éds), Le conditionnel en français, Université de Metz : Recherches linguistiques 25, 4566.

Gosselin, L., 2005, Temporalité et modalité, Bruxelles, Duculot-de Boeck.

Gosselin, L., 2010, Les modalités en français, Amsterdam-New York, Rodopi.

Gosselin, L., 2013, Semantic and Pragmatic Aspects of the Interaction of Time and Modality in French: an Interval-based Account, in Jaszczolt, K. et de Saussure, L. (éds), Time: Language, Cognition, and Reality, Oxford, OUP, 98-127.

Gosselin, L., 2014, Sémantique des jugements épistémiques, Langages 191, 63-81.

Gosselin, L., 2017, Les temps verbaux du français : du système au modèle, Verbum XXXIV, 31-69.

Imbs, P., 1960, L'emploi des temps verbaux en français moderne, Paris, Klincksieck.

Korzen, H., et Nølke, H., 1990, Projet pour une théorie des emplois du conditionnel, in O. Halmøy, A. Halvorsen et L. Lorentzen (éds) : Actes du onzième congrès des romanistes scandinaves, Université de Trondheim, 273-300.

Korzen, H., et Nølke, H., 2001, Le conditionnel : niveaux de modélisation, in Dendale P. et Tasmowski L. (éds.), Le conditionnel en français, Université de Metz : Recherches linguistiques 25, 125-146.

Kratzer, A., 1986, Conditionals, Chicago Linguistics Society 22(2), 1-15.

Martin, R., 1981, Le futur linguistique : temps linéaire ou temps ramifié ? (à propos du futur et du conditionnel en français), Langages 64, 81-92.

Martinez, M. 2011, Travelling in Branching Time, Disputatio IV, 31, 271-287.

Nilsson-Ehle, H., 1943, Le conditionnel «futur du passé» et la périphrase devait + infinitif, Studia Neophilologica, 16-1, 50-88.

Thomason, R., 1970, Indeterminist Time and Truth-value Gaps, Theoria 36(3), 264-281.

Touratier, C., 1996, Le système verbal français, Paris, Armand Colin.

Von Wright, G. H., 1984, Truth, Knowledge and Modality, Oxford, Basil Blackwell.

Vuillaume, M., 2001, L'expression du futur du passé en français et en allemand, in Dendale P. et Tasmowski L. (éds.), Le conditionnel en français, Université de Metz : Recherches linguistiques 25, 105-124.

Vuillemin, J., 1984, Nécessité ou contingence. L'aporie de Diodore et les systèmes philosophiques, Paris, Minuit.

Wilmet, M., 2001, L'architectonique du conditionnel, in P. Dendale et L. Tasmowski (éds.), Le conditionnel en français, Université de Metz : Recherches linguistiques 25, 21-44. 